\title{
METRIZATION OF PROXIMITY SPACES ${ }^{1}$
}

\author{
SOLOMON LEADER
}

0 . Introduction. Our purpose here is to present a metrization criterion for proximity spaces that is analogous to R. L. Moore's criterion for topological spaces [8]. Where Moore's criterion demands a sequence of open coverings, our criterion requires "admissible" coverings. In Moore's criterion a set $A$ is close to a point $b$ (that is, $b \in \bar{A}$ ) if and only if the star of $A$ meets the star of $b$ for every covering in the sequence. For proximity spaces we require only that a set $A$ be close to a set $B$ if and only if the star of $A$ meets $B$ for every covering in the sequence. Such weakening of the star-separation condition is possible because proximity spaces have a strong separation axiom. However, we must require that our sequence of coverings be nested by refinement.

Our covering criterion is easily translated into a criterion involving "admissible" entourages. These entourages play a key role in the criterion of Efremovič and Švarc [6]. Their criterion easily implies ours, but the converse is much more difficult. It should therefore be easier to prove a given proximity space metrizable using our criterion rather than that of [6].

1. The Metrization Theorem. Let $(X, \beta)$ be a proximity space in the sense of Efremovič [3]-[5]. We recall some basic definitions. $B$ is a $\beta$-neighborhood of $A$ whenever $A \not \beta X-B .(X, \beta)$ is metrizable if there exists a metric $\sigma$ on $X$ such that for all subsets $A, B$ of $X$

(1) $A \beta B$ if and only if $\sigma(A, B)=\inf \{\sigma(a, b):(a, b) \in A \times B\}=0$.

A pseudometric $\sigma$ on $X$ is a $\beta$-gauge [10] if the direct implication holds in (1).

We shall call a subset $P$ of the cartesian product $X^{2}$ compressed if $P$ is infinite and $A \beta B$ for every pair of subsets $A, B$ of $X$ with $(A \times B) \cap P$ infinite. A covering $U$ of $X$ is admissible if for every compressed set $P$ there exist $(x, y)$ in $P$ and $E$ in $U$ such that both $x$ and $y$ belong to $E$. An entourage (subset of $X^{2}$ containing the diagonal $I$ ) $U$ is admissible if $U$ meets every compressed subset $P$ of $X^{2}$. Since every infinite subset of a compressed set is compressed, $U$ must contain almost all (all but finitely many points) of $P$.

We can now state the Metrization Theorem. Its proof will be given in $\$ 5$.

Received by the editors September 23, 1966.

${ }^{1}$ Work done under NSF GP-4413. 
THEOREM I. For any proximity space $(X, \beta)$ the following three conditions are equivalent:

(i) $(X, \beta)$ is metrizable.

(ii) There exists a sequence $\left\{U_{n}\right\}$ of admissible coverings with $U_{n+1}$ a refinement of $U_{n}$ and

(2) $A \beta B$ if and only if for every $n$ some member of $U_{n}$ meets both $A$ and $B$.

(iii) There exists a sequence $\left\{U_{n}\right\}$ of admissible symmetric entourages with $U_{n+1} \subseteq U_{n}$ and

$$
A \beta B \text { if and only if } A \times B \text { meets } U_{n} \text { for all } n \text {. }
$$

2. Equivalent sequences. An entourage $U$ is a $\beta$-entourage if $U[A]$ is a $\beta$-neighborhood of $A$ for every subset $A$ of $X$. An entourage $U$ is simple if it is the complement of a product:

$$
U=X^{2}-A \times B .
$$

Clearly, the entourage (4) is a $\beta$-entourage if and only if $A \not \beta B$.

We can now characterize the equivalence relation $\left\{x_{n}\right\} \sim\left\{y_{n}\right\}$ introduced in [6].

Theorem II. For any sequence $\left\{\left(x_{n}, y_{n}\right)\right\}$ in $X^{2}$ the following seven conditions are equivalent:

(i) For every infinite set $M$ of positive integers $\left\{x_{n}: n \in M\right\}$ $\beta\left\{y_{n}: n \in M\right\}$.

(ii) If $\left(x_{n}, y_{n}\right) \in A \times B$ for infinitely many $n$, then $A \beta B$.

(iii) $\operatorname{Lim}_{n \rightarrow \infty} \sigma\left(x_{n}, y_{n}\right)=0$ for every $\beta$-gauge $\sigma$.

(iv) $\operatorname{Lim}_{n \rightarrow \infty} \sigma\left(x_{n}, y_{n}\right)=0$ for every totally bounded $\beta$-gauge $\sigma$.

(v) Given any simple $\beta$-entourage $U,\left(x_{n}, y_{n}\right) \in U$ for almost all $n$.

(vi) Given any entourage $U$ in the precompact uniform structure associated [1], [7] with $\beta,\left(x_{n}, y_{n}\right) \in U$ for almost all $n$.

(vii) Given any entourage $U$ in the total structure associated [2] with $\beta,\left(x_{n}, y_{n}\right) \in U$ for almost all $n$.

Proof. The equivalence of (i) and (ii) is trivial. The equivalence of (ii) and (iii) is just part (b) of Lemma 3 in [10]. (iii) is equivalent to (viii) because the total $\beta$-structure is generated by the $\beta$-gauges $[2],[11]$. Analogously, (iv) is equivalent to (vi) because the precompact $\beta$-structure is generated by the totally bounded $\beta$-gauges [11]. (v) is equivalent to (vi) because the simple $\beta$-entourages form a subbase for the precompact $\beta$-structure [1], [7]. (iii) implies (iv) a fortiori. Finally, (iv) implies (ii) because $A \beta B$ if and only if $\sigma(A, B)=0$ for every totally bounded $\beta$-gauge [5], [10]. 


\section{Compressed sets.}

Theorem III. For any infinite subset $P$ of $X^{2}$ the following eight conditions are equivalent:

(o) $P$ is compressed.

(i) If $Q$ is any infinite subset of $P$, then for $\pi_{j}\left(x_{1}, x_{2}\right)=x_{j}$ with $j=1,2, \pi_{1} Q \beta \pi_{2} Q$.

(ii) If $\left\{\left(x_{n}, y_{n}\right)\right\}$ is any sequence of distinct points in $P$, then $\left\{x_{n}\right\} \sim\left\{y_{n}\right\}$.

(iii) Given any $\beta$-gauge $\sigma$ and $\epsilon>0$,

$$
\sigma(x, y) \leqq \epsilon \text { for almost all }(x, y) \text { in } P \text {. }
$$

(iv) Given any totally bounded $\beta$-gauge $\sigma$ and $\epsilon>0$, (5) holds.

(v) Every simple $\beta$-entourage (4) contains almost all of $P$.

(vi) Every entourage in the precompact $\beta$-structure contains almost all of $P$.

(vii) Every entourage in the total $\beta$-structure contains almost all of $P$.

Proof. Defining $A=\pi_{1} Q$ and $B=\pi_{2} Q$ we have $Q \subseteq(A \times B) \cap P$. Hence (o) implies (i). To prove the converse apply (i) with $Q=(A \times B) \cap P$ to get $\pi_{1} Q \subseteq A$ and $\pi_{2} Q \subseteq B$. Then, for $Q$ infinite, (i) implies $A \beta B$, hence (o).

To prove (i) implies (ii) apply (i) with $Q=\left\{\left(x_{n}, y_{n}\right): n \in M\right\}$ to get (i) of Theorem II. The converse follows similarly by applying (ii) to a sequence of distinct points in $Q$.

The equivalence of (ii) with each of the conditions (iii) -(vii) follows from Theorem II using the respective characterizations (iii)(vii) of sequential equivalence.

4. Admissible entourages. We next show that the admissible entourages are precisely those introduced in [6].

TheOREM IV. An entourage $U$ is admissible if and only if for every pair of equivalent sequences $\left\{x_{n}\right\} \sim\left\{y_{n}\right\}$ in $X,\left(x_{n}, y_{n}\right) \in U$ for some (hence, almost all) n.

Proof. Let $U$ be admissible and $\left\{x_{n}\right\} \sim\left\{y_{n}\right\}$. Let $P$ be the range of the double sequence $\left\{\left(x_{n}, y_{n}\right)\right\}$. We contend $U$ meets $P$.

If $P$ is finite, then the double sequence has a constant subsequence. By (i) of Theorem II a constant subsequence must lie in the diagonal $I$ since $X$ is Hausdorff. Since $U$ contains $I, U$ meets $P$.

On the other hand, if $P$ is infinite then, according to (i) of Theorems II and III, $P$ is compressed. Hence, $U$ meets $P$. 
Conversely, let an entourage $U$ satisfy the sequential condition. Given any compressed set $P$, choose a sequence $\left\{\left(x_{n}, y_{n}\right)\right\}$ of distinct points in $P$ to conclude from (ii) of Theorem III that $\left\{x_{n}\right\} \sim\left\{y_{n}\right\}$. Hence $\left(x_{n}, y_{n}\right) \in U$ for some $n$. So $U$ meets $P$ and is therefore admissible.

5. Proof of Theorem I. Recall that every covering $U$ induces a symmetric entourage $U$, namely

$$
U=U\left\{E^{2}: E \in U\right\} .
$$

Comparison of our definitions immediately shows that under (6) $U$ is an admissible entourage if and only if $U$ is an admissible covering.

Given (i) of Theorem I let $\sigma$ be a metric for $(X, \beta)$. Let $U_{n}$ be the covering consisting of all subsets of $X$ with $\sigma$-diameter at most $1 / n$. Then (2) follows immediately from (1). Moreover, $U_{n+1}$ is a refinement of $U_{n}$ since it is actually a subcovering. To show that each $U_{n}$ is an admissible covering we need only note that the entourage $U_{n}=\sigma^{-1}[0,1 / n]$ induced by (6) is admissible because (o) implies (iii) in Theorem III. Hence (i) implies (ii).

To prove (ii) implies (iii) let $U_{n}$ be the admissible symmetric entourage (6) induced by the admissible covering $U_{n}$. Then (2) translates via (6) into (3). Moreover, since $U_{n+1}$ refines $U_{n}, U_{n+1} \subseteq U_{n}$.

To prove (iii) implies (i) it suffices to prove, in view of (1), (3), and the Metrization Lemma [9], that $\left\{U_{n}\right\}$ is a base for a uniform structure. That is, we must show that for each $m$ there exists $n$ with $U_{n}^{2} \subseteq U_{m}$. Suppose this were false. Then there would exist $m$ such that for all $n$ we could choose $\left(x_{n}, y_{n}\right)$ in $U_{n}^{2}-U_{m}$. We could thereby also choose $z_{n}$ for all $n$ such that both $\left(x_{n}, z_{n}\right)$ and $\left(z_{n}, y_{n}\right)$ belong to $U_{n}$, while

$$
\left(x_{n}, y_{n}\right) \notin U_{m} .
$$

Applying (3) to get (ii) of Theorem II, we conclude that $\left\{x_{n}\right\} \sim\left\{z_{n}\right\}$ and $\left\{z_{n}\right\} \sim\left\{y_{n}\right\}$. Hence, by (iii) of Theorem II and the triangle inequality, $\left\{x_{n}\right\} \sim\left\{y_{n}\right\}$ which according to Theorem IV contradicts (7).

\section{REFERENCES}

1. E. M. Alfsen and J. E. Fenstad, On the equivalence between proximity structures and totally bounded uniform structures, Math. Scand. 7 (1959), 353-360.

2. E. M. Alfsen and O. Njåstad, Proximity and generalized uniformity, Fund. Math. 52 (1963), 235-252.

3. V. A. Efremovič, Infinitesimal spaces, Dokl. Akad. Nauk SSSR 76 (1951), $341-343$. 
4. —_ Infinitesimal spaces, Uspehi Mat. Nauk 6 (1951), no. 4 (44), 203-204.

5. - The geometry of proximity, Mat. Sb. 31 (73) (1952), 189-200.

6. V. A. Efremovič and A. S. Svarc, $A$ new definition of uniform spaces. Metrization of proximity spaces. Dokl. Akad. Nauk SSSR 89 (1953), 393-396.

7. I. S. Gál, Proximity relations and precompact structures, Nederl. Akad. Wetensch. Proc. Ser. A 62 = Indag. Math. 21 (1959), 304-326.

8. F. B. Jones, Metrization, Amer. Math. Monthly (73) 6 (1966), 571-576.

9. J. L. Kelley, General topology, Van Nostrand, Princeton, N. J., 1955.

10. S. Leader, On completion of proximity spaces by local clusters, Fund. Math. 48 (1960), 201-216.

11. On pseudometrics for generalized uniform structures, Proc. Amer. Math. Soc. 16 (1965), 493-495.

Rutgers-The State University 